\title{
ON CONVEX COMBINATIONS OF TWO VALUES
}

Abstract. We study values for cooperative TU-games which are convex combinations of the Shapley value and the solidarity value, introduced in our recent paper [1]. First, we axiomatize the convex combination of the two values in the case when the coefficients are given exogenously. Next, we give an axiomatic description of the whole family of such values.

1. Introduction. Let $N$ be a finite set of $n$ players, called the grand coalition. For any coalition $T \subseteq N$, the small letter $t$ will generically denote the cardinality of $T$. To simplify the notation one-member coalitions, for example $\{i\}$, will sometimes be denoted by $i$.

Let $\Gamma$ denote the linear space of all $n$-person transferable utility (TU) games. A value on $\Gamma$ is thought of as a vector-valued mapping, say $\varphi: \Gamma \rightarrow$ $\mathbb{R}^{n}$, which uniquely determines, for each $v \in \Gamma$, a distribution of the wealth available to the players through their participation in the game $v$.

The famous Shapley value $\Phi^{\mathrm{Sh}}$ on $\Gamma$ is given by the formula

$$
\Phi_{i}^{\mathrm{Sh}}(v)=\sum_{T \ni i} \frac{(n-t) !(t-1) !}{n !}[v(T)-v(T \backslash i)], \quad i \in N, v \in \Gamma .
$$

In our recent paper [1], we introduced another value for TU-games, called the solidarity value. Let $T$ be a non-empty coalition and let $v \in \Gamma$. The quantity

$$
A^{v}(T):=\sum_{k \in T}[v(T)-v(T \backslash k)] / t
$$

is the average marginal contribution of a member of the coalition $T$. The solidarity value $\Phi^{\mathrm{Sol}}$ on $\Gamma$ is defined by

1991 Mathematics Subject Classification: 90D12, 90D06.

Key words and phrases: cooperative transferable utility games, Shapley value, average values. 


$$
\Phi_{i}^{\mathrm{Sol}}(v)=\sum_{T \ni i} \frac{(n-t) !(t-1) !}{n !} A^{v}(T), \quad i \in N, v \in \Gamma .
$$

To illustrate the difference between these two values, we give a simple example.

Example. Let $N=\{1,2\}$. Assume that $v(N)=3, v(1)=1$, and $v(2)=$ 0 . The Shapley value for this game is $\Phi^{\mathrm{Sh}}(v)=(2,1)$ while the solidarity value for $v$ is $\Phi^{\mathrm{Sol}}(v)=(1.75,1.25)$. If the solidarity value is accepted by the players as a solution for this game then one can say that player 1 seems to be quite generous for player 2, because his or her "bargaining position" in this game is much stronger than that of player 2 . On the other hand, it is interesting to note that the ratio of the marginal contributions to the grand coalition of players 1 and 2 equals $(v(N)-v(2)) /(v(N)-v(1))=1.5$ and is much closer to $\Phi_{1}^{\mathrm{Sol}}(v) / \Phi_{2}^{\mathrm{Sol}}(v)=1.4$ than to $\Phi_{1}^{\mathrm{Sh}}(v) / \Phi_{2}^{\mathrm{Sh}}(v)=2$.

This example shows that the solidarity value can be accepted by the players as a solution of the game if there are some friendly relations between them which are not reflected by the characteristic function itself. The solidarity value does not seem to be a very good solution concept for studying pure economic models. However, when we think of some real social or political situations (or conflicts) sometimes we may have an impression that (at least in some subcoalitions) people would be willing to accept the solidarity value.

The Shapley value $\Phi^{\mathrm{Sh}}$ does not benefit null players in any game $v$. (By definition, $i \in N$ is a null player if $v(S \cup i)=v(S)$ for any coalition $S \subset N \backslash i$.) According to $\Phi^{\mathrm{Sh}}$ every null player in $v$ gets nothing in this game. Thus, the Shapley value represents a rather severe (from social point of view) solution concept. On the other hand, the solidarity value $\Phi^{\text {Sol }}$ represents a pretty strong social approach. Namely, a null player in $v$ can be rewarded by $\Phi^{\text {Sol }}$ with a positive payoff, at least in some games. Therefore, to diminish the "severity" of $\Phi^{\mathrm{Sh}}$ as well as the strong social properties of $\Phi^{\mathrm{Sol}}$, it seems reasonable to consider compromise values between the Shapley value and the solidarity value. In this paper, we study such values in the form of convex combinations of these two values. An example is the average value given by

$$
\left[\Phi^{\mathrm{Sh}}(v)+\Phi^{\mathrm{Sol}}(v)\right] / 2, \quad v \in \Gamma .
$$

2. Average values. Let $\varphi$ be a value on $\Gamma$. We adopt the following standard axioms:

Axiom A1 (Efficiency). For any game $v \in \Gamma, \sum_{i \in N} \varphi_{i}(v)=v(N)$.

Axiom A2 (Additivity). For any games $v, w \in \Gamma, \varphi(v+w)=\varphi(v)+\varphi(w)$.

Axiom A3 (Symmetry). Let $v \in \Gamma$. For any automorphism $\pi$ of the game $v, \varphi_{i}(v)=\varphi_{\pi(i)}(v)$. 
We remind that $\pi$ is an automorphism of the game $v$ if $v(\pi(S))=v(S)$ for each coalition $S \subset N$.

The next postulate was introduced in [1].

Axiom A4 ( $A$-null player). If $i \in N$ is an $A$-null player in a game $v \in \Gamma$, that is, $A^{v}(T)=0$ for every coalition $T$ containing $i$, then $\varphi_{i}(v)=0$.

If every coalition $T$ including player $i$ has the average marginal contribution $A^{v}(T)=0$, then according to A4 player $i$ gets nothing from the game $v$. As shown in [1], Axioms A1-A4 uniquely determine the solidarity value.

We now introduce a new definition and an axiom which enables us to derive the average values mentioned above.

DeFInItion 2.1. A player $i \in N$ has a reverse contributions property in a game $v \in \Gamma$ if for each coalition $S$ including player $i$,

$$
v(S)-v(S \backslash i)=-A^{v}(S):=-\frac{1}{s} \sum_{k \in S}[v(S)-v(S \backslash k)] .
$$

AxIOM A5 (Reverse contributions). If $i \in N$ has the reverse contributions property in a game $v \in \Gamma$, then $\varphi_{i}(v)=0$.

In other words, if the marginal contribution of player $i$ to any coalition $S$ is the negative of the average contribution of the members of $S$, then such a player gets nothing from the game. We have the following result.

THEOREM 2.1. There exists a unique value $\varphi$ on $\Gamma$ satisfying Axioms $\mathrm{A} 1-\mathrm{A} 3$ and $\mathrm{A} 5$ and it is of the form

$$
\varphi_{i}(v)=\frac{1}{2} \Phi_{i}^{\mathrm{Sh}}(v)+\frac{1}{2} \Phi_{i}^{\mathrm{Sol}}(v), \quad i \in N, v \in \Gamma .
$$

This result is a special case of a more general theorem which we will prove in Section 3.

3. A generalization. In this section, we present a natural generalization of Axiom 5, involving some non-negative parameter $\alpha$.

Definition 3.1. Let $\alpha \geq 0$ be fixed. Player $i$ is said to have the $\alpha$-reverse contributions property in a game $v \in \Gamma$ if for each coalition $S$ including player $i$,

$$
v(S)-v(S \backslash i)=-\alpha A^{v}(S):=-\alpha \frac{1}{s} \sum_{k \in S}[v(S)-v(S \backslash k)] .
$$

Now we are ready to propose the next axiom. According to the above definition, player $i$ has the $\alpha$-reverse contributions property when his marginal contribution to any coalition $S$ is the negative of the average contribution of the members of $S$, up to the coefficient $\alpha$. Therefore, it is very natural to write Axiom A5 in a more general form as follows. 
Axiom A6 ( $\alpha$-reverse contributions). If $i \in N$ has the $\alpha$-reverse contributions property in a game $v \in \Gamma$, then $\varphi_{i}(v)=0$.

We can now state a generalization of Theorem 2.1.

TheOREM 3.1. Let $\alpha \geq 0$. There exists a unique value $\Phi^{\lambda}$ on $\Gamma$ satisfying Axioms $\mathrm{A} 1-\mathrm{A} 3$ and $\mathrm{A} 6$ and it is of the form

$$
\Phi_{i}^{\lambda}(v)=\lambda \Phi_{i}^{\mathrm{Sh}}(v)+(1-\lambda) \Phi_{i}^{\mathrm{Sol}}(v) \quad \text { for } i \in N, v \in \Gamma,
$$

where $\lambda=1 /(1+\alpha)>0$.

Throughout the rest of this section, let $\alpha \geq 0$ be fixed arbitrarily.

To prove Theorem 3.1, we introduce a special basis for the linear space $\Gamma$.

Definition 3.2. Let $\lambda=1 /(1+\alpha)$. For each $T \subset N, T \neq \emptyset$, we define the game $w_{T}$ by

$$
w_{T}(S)= \begin{cases}\frac{t !}{s !} \prod_{j=1}^{s-t}(\lambda t+j) & \text { if } S \supset T, \\ 0 & \text { otherwise }\end{cases}
$$

assuming that $\prod_{j=1}^{0}(\lambda t+j)=1$. In particular, $w_{T}(T):=1$.

LEMMA 3.1. The family $\left\{w_{T}: T \subset N, T \neq \emptyset\right\}$ of games defined by (3.3) is a basis for the linear space $\Gamma$.

Proof. The proof is similar to that of Lemma 2.2 in [1].

LEMMA 3.2. Let $T$ be any non-empty coalition such that $T \neq N$, and let $S=T \cup D$ where $\emptyset \neq D \subseteq N \backslash T$. Then, for every player $i \in S \backslash T$, we have

$$
(1+\alpha) w_{T}(S)=w_{T}(S \backslash i)+\alpha \frac{1}{s} \sum_{k \in S} w_{T}(S \backslash k)
$$

and, moreover, every player $i \in N \backslash T$ has the $\alpha$-reverse contributions property in the game $w_{T}$.

P r o of. Assume first that $d \geq 2$, where $d$ is the cardinality of $D$. Using (3.3), we get

$$
\begin{aligned}
(1+\alpha) w_{T}(S)-w_{T}(S \backslash i)-\alpha \frac{1}{s} \sum_{k \in S} w_{T}(S \backslash k) \\
=(1+\alpha) \frac{t !}{s !} \prod_{j=1}^{s-t}(\lambda t+j)-w_{T}(S \backslash i)-\alpha \frac{1}{s} \sum_{k \in D} w_{T}(S \backslash k) \\
=(1+\alpha) \frac{t !}{(t+d) !} \prod_{j=1}^{d}(\lambda t+j)-\frac{t !}{(t+d-1) !} \prod_{j=1}^{d-1}(\lambda t+j)
\end{aligned}
$$




$$
\begin{aligned}
& -\alpha \frac{d}{(t+d)} \frac{t !}{(t+d-1) !} \prod_{j=1}^{d-1}(\lambda t+j) \\
= & \frac{t !}{(t+d) !} \prod_{j=1}^{d-1}(\lambda t+j)[(1+\alpha)(\lambda t+d)-(t+d)-\alpha d]=0,
\end{aligned}
$$

because $\lambda=1 /(1+\alpha)$. Thus (3.4) follows for $d \geq 2$. If $d=1$, then the above calculations also prove (3.4) if we put $\prod_{j=1}^{d-1}(\lambda t+j)=1$.

Consider now any player $i \in N \backslash T$. Let $S$ be an arbitrary coalition including player $i$. If $T$ is not a subset of $S$, then both sides of equation (3.1) with $v=w_{T}$ are equal to zero. If $T$ is a subset of $S$, then $i \in S \backslash T$, and equation (3.1) with $v=w_{T}$ is readily equivalent to (3.4), which has already been established. Thus, player $i$ has the $\alpha$-reverse contributions property.

Lemma 3.3. If $\varphi$ is a value on $\Gamma$ satisfying Axioms $\mathrm{A} 1-\mathrm{A} 3$ and $\mathrm{A} 6$, then for each non-empty coalition $T \neq N$, and any real number $c$, we have

$$
\varphi_{i}\left(c w_{T}\right)= \begin{cases}\frac{c(t-1) !}{n !} \prod_{j=1}^{n-t}(\lambda t+j) & \text { if } i \in T, \\ 0 & \text { if } i \notin T,\end{cases}
$$

and if $T=N$, then $\varphi_{i}\left(c w_{T}\right)=c / n$ for each $i \in N$.

Proof. Fix any non-empty coalition $T \neq N$. If $c=0$, then the lemma follows immediately from Axioms A1 and A2. Assume that $c \neq 0$. From Lemma 2.2, we easily conclude that every player $i \in N \backslash T$ has the $\alpha$-reverse contributions property in the game $c w_{T}$. By Axiom A6, $\varphi_{i}\left(c w_{T}\right)=0$ for all $i \in N \backslash T$, and the remaining part of (3.5) follows now from (3.3) and the efficiency and symmetry axioms. If $T=N$, the proof is trivial.

From A2 and Lemmas 3.1 and 3.3, we deduce the following simple fact.

Lemma 3.4. Any value satisfying axioms $\mathrm{A} 1-\mathrm{A} 3$ and $\mathrm{A} 6$ is a linear mapping from $\Gamma$ into $\mathbb{R}^{n}$.

Proof of Theorem 3.1. First, we show the existence of a value satisfying our Axioms A1-A3 and A6. Clearly, $\Phi^{\lambda}$ given by (3.2) satisfies Axioms A2-A3. $\Phi^{\lambda}$ also satisfies A1 since both the Shapley and the solidarity values are efficient [1], [2]. To prove that $\Phi^{\lambda}$ also satisfies A6 with $\alpha=(1-\lambda) / \lambda \geq 0$, note that

$$
\begin{aligned}
\Phi_{i}^{\lambda}(v) & =\sum_{S \ni i} \frac{(n-s) !(s-1) !}{n !}\left(\lambda[v(S)-v(S \backslash i)]+(1-\lambda) A^{v}(S)\right) \\
& =\sum_{S \ni i} \frac{(n-s) !(s-1) !}{n !(1+\alpha)}\left(v(S)-v(S \backslash i)+\alpha A^{v}(S)\right)
\end{aligned}
$$


for any $v \in \Gamma, i \in N$. Thus, if player $i$ has the $\alpha$-reverse contributions property in a game $v$, then it follows from (3.6) that $\Phi_{i}^{\lambda}(v)=0$, that is, $\Phi^{\lambda}$ satisfies our assumption A6.

To prove the uniqueness, consider a value $\varphi$ on $\Gamma$ which satisfies A1-A3 and A6. By Lemma 3.4, $\varphi$ is a linear mapping. Clearly, the value $\Phi^{\lambda}$ is also linear. Applying Lemma 3.3 to both values $\varphi$ and $\Phi^{\lambda}$, we conclude that $\varphi\left(w_{T}\right)=\Phi^{\lambda}\left(w_{T}\right)$, for each base game $w_{T}$ defined by (3.3). Thus, $\varphi(v)=\Phi^{\lambda}(v)$ for every $v \in \Gamma$.

4. Axiomatization of the class of values $\left\{\Phi^{\lambda}: \lambda \in(0,1]\right\}$. In this section we do not assume that $\lambda$ (or equivalently $\alpha$ ) is exogenously given and describe axiomatically the class of all possible values of the form given in Theorem 3.1. We first fix some notation and state an auxiliary lemma.

For $i \in N$, let $\Gamma_{0}(i)$ be the linear space of all games $v \in \Gamma$ in which $i$ is a null player, and let $\Gamma_{1}(i)$ be the linear space of all games $v \in \Gamma$ in which $i$ is an $A$-null player. Further, we put $\Gamma(i):=\{v \in \Gamma: v(i)=0\}$.

Lemma 4.1. For each $i \in N, \Gamma(i)$ is the direct sum of the linear spaces $\Gamma_{0}(i)$ and $\Gamma_{1}(i)$, that is,

$$
\Gamma(i)=\Gamma_{0}(i)+\Gamma_{1}(i)
$$

and

$$
\Gamma_{0}(i) \cap \Gamma_{1}(i)=\{0\} .
$$

Proof. Fix $i \in N$. To prove (4.2), let $v \in \Gamma_{0}(i) \cap \Gamma_{1}(i)$. Then, for each coalition $S \subseteq N \backslash i$, we have

$$
v(S \cup i)=v(S) \quad \text { and } \quad \sum_{k \in S \cup i}[v(S \cup i)-v((S \cup i) \backslash k)]=0 .
$$

Hence, we have

$$
\sum_{k \in S}[v(S \cup i)-v((S \cup i) \backslash k)]=0 .
$$

If we put $S=\{j\}$ in (4.3), where $j \neq i$, we get $v(\{i, j\})=v(i)$. Since $v \in \Gamma_{0}(i)$, we have $v(i)=0$ and, moreover, $v(\{i, j\})=v(j)$. Thus, we get $v(j)=v(\{i, j\})=v(i)=0$. Similarly, by induction on the cardinality of $S$, we can show that $v(S)=v(S \cup i)=0$ for each $S \subseteq N \backslash i$. In other words, $v$ is the null game, and (4.2) easily follows.

Note that $\Gamma_{0}(i) \subset \Gamma(i), \Gamma_{1}(i) \subset \Gamma(i)$, and that the dimension of the space $\Gamma(i)$ equals $2^{n}-2$. To prove (4.1), it now suffices to show that both $\Gamma_{0}(i)$ and $\Gamma_{1}(i)$ are at least $\left(2^{n-1}-1\right)$-dimensional linear spaces. 
Define the following two classes of games:

$$
\begin{aligned}
& B_{0}(i)=\left\{u_{T}: i \notin T \text { and } T \neq \emptyset\right\}, \\
& B_{1}(i)=\left\{w_{T}: i \notin T \text { and } T \neq \emptyset\right\},
\end{aligned}
$$

where

$$
u_{T}(S)= \begin{cases}1 & \text { if } S=T \text { or } S=T \cup i \\ 0 & \text { otherwise }\end{cases}
$$

and $w_{T}$ are the base games $(3.3)$.

One can easily check that $B_{0}(i)$ is a set of linearly independent games. Similarly, by Lemma 3.1, all games in $B_{1}(i)$ are linearly independent. On the other hand, we can directly verify that $B_{0}(i) \subset \Gamma_{0}(i)$, and from Lemma 3.2, it follows that $B_{1}(i) \subset \Gamma_{1}(i)$. The cardinality of $B_{0}(i)$, and also of $B_{1}(i)$, is equal to $2^{n-1}-1$. Thus, $\Gamma_{0}(i)$ and $\Gamma_{1}(i)$ are at least $\left(2^{n-1}-1\right)$-dimensional linear spaces and the lemma follows.

In order to axiomatize the above mentioned class of values, we now give our new axioms. It is natural to expect that the "extreme points" $\Phi^{\text {Sh }}$ and $\Phi^{\mathrm{Sol}}$ in the set of values of our interest will play an important role in our approach.

Axiom A7 (Shapley value proportionality). Suppose that $i \in N$ is an $A$-null player in two games $v$ and $w$. Then

$$
\Phi_{i}(v) \Phi_{i}^{\mathrm{Sh}}(w)=\Phi_{i}(w) \Phi_{i}^{\mathrm{Sh}}(v) .
$$

The next axiom is simply symmetric to the previous one.

AxIOM A8 (Solidarity value proportionality). Suppose that $i \in N$ is a null player in two games $v$ and $w$. Then

$$
\Phi_{i}(v) \Phi_{i}^{\mathrm{Sol}}(w)=\Phi_{i}(w) \Phi_{i}^{\mathrm{Sol}}(v) .
$$

AxIOM A9 (Rationality). If for some $i \in N, v \in \Gamma$ and for each coalition $S$ including player $i$, we have

$$
v(S)-v(S \backslash i) \geq 0 \quad \text { and } \quad A^{v}(S):=\frac{1}{s} \sum_{k \in S}[v(S)-v(S \backslash k)] \geq 0,
$$

then $\Phi_{i}(v) \geq 0$.

The conclusion of Axiom A7 can be written in the following more readable way:

$$
\Phi_{i}(v) / \Phi_{i}(w)=\Phi_{i}^{\mathrm{Sh}}(v) / \Phi_{i}^{\mathrm{Sh}}(w)
$$

provided that we know that both $\Phi_{i}(w)$ and $\Phi_{i}^{\mathrm{Sh}}(w)$ are different from zero. A similar remark concerns Axiom A8.

Here is our second main result in this paper. 
Theorem 4.1. A value $\Phi$ on $\Gamma$ satisfies Axioms $\mathrm{A} 1-\mathrm{A} 3$ and $\mathrm{A} 7-\mathrm{A} 9$ if and only if there exists some $\alpha \geq 0$ such that $\Phi=\Phi^{\lambda}$ described in Theorem 3.1 with $\lambda=1 /(1+\alpha)$.

Proof. The implication $(\Leftarrow)$ is obvious. The proof of $(\Rightarrow)$ consists of three steps.

S t ep 1 . We first prove that for every $i \in N$ there exists a real number $\lambda_{i}$ such that

$$
\Phi_{i}(v)=\lambda_{i} \Phi_{i}^{\mathrm{Sh}}(v)+\left(1-\lambda_{i}\right) \Phi_{i}^{\mathrm{Sol}}(v) \quad \text { for all } v \in \Gamma .
$$

For this, fix an $i \in N$, recall (4.4), and choose any $w \in B_{1}(i) \subset \Gamma_{1}(i)$ such that $\Phi_{i}^{\mathrm{Sh}}(w)>0$. Put $\lambda_{i}=\Phi_{i}(w) / \Phi_{i}^{\mathrm{Sh}}(w)$. By Axiom A7, we have

$$
\Phi_{i}(v)=\lambda_{i} \Phi_{i}^{\mathrm{Sh}}(v) \quad \text { for all } v \in \Gamma_{1}(i) .
$$

Similarly, choose any $u \in B_{0}(i) \subset \Gamma_{0}(i)$ with $\Phi_{i}^{\text {Sol }}(w) \neq 0$ and put $\gamma_{i}=$ $\Phi_{i}(u) / \Phi_{i}^{\text {Sol }}(u)$. By Axiom A8, we have

$$
\Phi_{i}(v)=\gamma_{i} \Phi_{i}^{\mathrm{Sol}}(v) \quad \text { for all } v \in \Gamma_{0}(i) .
$$

On the other hand, we know that

$$
\Phi_{i}^{\mathrm{Sh}}(v)=0 \quad \text { for all } v \in \Gamma_{0}(i),
$$

and

$$
\Phi_{i}^{\mathrm{Sol}}(v)=0 \quad \text { for all } v \in \Gamma_{1}(i) .
$$

Using (4.6)-(4.9) and Lemma 4.1, we infer that

$$
\Phi_{i}(v)=\lambda_{i} \Phi_{i}^{\mathrm{Sh}}(v)+\gamma_{i} \Phi_{i}^{\mathrm{Sol}}(v) \quad \text { for all } v \in \Gamma(i) .
$$

Consider now the game $w$ in $\Gamma(i)$ described by: $w(S)=n$ if $S=N$, and $w(S)=0$ otherwise. The efficiency and symmetry axioms A1 and A3 imply immediately that $\Phi_{i}(w)=1$. Similarly, $\Phi_{i}^{\mathrm{Sh}}(w)=\Phi_{i}^{\mathrm{Sol}}(w)=1$. Substituting $v=w$ in (4.10), we get $\gamma_{i}=1-\lambda_{i}$, and consequently (4.5) holds for each $v \in \Gamma(i)$.

Consider now the game $u$ defined as follows: $u(\emptyset)=0$ and $u(S)=1$ for all $S \neq \emptyset$. Of course, $u \notin \Gamma(i)$. Taking into account the fact that $\Gamma$ and $\Gamma(i)$ are $\left(2^{n}-1\right)$ - and $\left(2^{n}-2\right)$-dimensional linear spaces, respectively, we conclude that any game $v$ in $\Gamma$ can be represented as a sum $v=\bar{v}+c u$ with some $\bar{v} \in \Gamma(i)$ and a scalar $c$. Using the additivity, efficiency and symmetry axioms, we get for any $v \in \Gamma$,

$$
\Phi_{i}(v)=\Phi_{i}(\bar{v})+\Phi_{i}(c u)=\lambda_{i} \Phi_{i}^{\mathrm{Sh}}(\bar{v})+\left(1-\lambda_{i}\right) \Phi_{i}^{\mathrm{Sol}}(\bar{v})+c / n .
$$

On the other hand, we have

$$
\lambda_{i} \Phi_{i}^{\mathrm{Sh}}(v)+\left(1-\lambda_{i}\right) \Phi_{i}^{\mathrm{Sol}}(v)=\lambda_{i} \Phi_{i}^{\mathrm{Sh}}(\bar{v})+\left(1-\lambda_{i}\right) \Phi_{i}^{\mathrm{Sol}}(\bar{v})+c / n .
$$

Thus (4.5) holds for any game $v \in \Gamma$. 
Step 2. Let $n \geq 2$. We now prove that $\lambda_{i}$ in (4.5) is independent of $i \in N$. Note that the efficiency axiom implies that

$$
\sum_{i \in N}\left[\lambda_{i} \Phi_{i}^{\mathrm{Sh}}(v)+\left(1-\lambda_{i}\right) \Phi_{i}^{\mathrm{Sol}}(v)\right]=v(N) \quad \text { for all } v \in \Gamma
$$

which, by the efficiency of $\Phi^{\mathrm{Sol}}$, is equivalent to

$$
\sum_{i \in N} \lambda_{i}\left[\Phi_{i}^{\mathrm{Sh}}(v)-\Phi_{i}^{\mathrm{Sol}}(v)\right]=0 \quad \text { for all } v \in \Gamma .
$$

This, in turn, because of the efficiency of $\Phi^{\mathrm{Sh}}$ and $\Phi^{\mathrm{Sol}}$, gives

$$
\sum_{i=2}^{n} \gamma_{i}\left[\Phi_{i}^{\mathrm{Sh}}(v)-\Phi_{i}^{\mathrm{Sol}}(v)\right]=0 \quad \text { for all } v \in \Gamma,
$$

where

$$
\gamma_{i}=\lambda_{i}-\lambda_{1} \quad \text { for } i=2, \ldots, n .
$$

Define the games $v_{k}(k=2, \ldots, n)$ in the following way:

$$
v_{k}(S)= \begin{cases}1 & \text { if } S=N \backslash k, \\ 0 & \text { otherwise. }\end{cases}
$$

Using (4.11), we get

$$
\sum_{i=2}^{n} \gamma_{i}\left[\Phi_{i}^{\mathrm{Sh}}\left(v_{k}\right)-\Phi_{i}^{\mathrm{Sol}}\left(v_{k}\right)\right]=0, \quad k=2, \ldots, n .
$$

At the same time, we have $\Phi_{k}^{\mathrm{Sh}}\left(v_{k}\right)=-1 / n, \Phi_{k}^{\mathrm{Sol}}\left(v_{k}\right)=-1 / n^{2}$ and $\Phi_{i}^{\mathrm{Sh}}\left(v_{k}\right)$ $=1 /(n-1) n, \Phi_{i}^{\mathrm{Sol}}\left(v_{k}\right)=1 /(n-1) n^{2}$ for all $i \neq k$. Hence, (4.13) reduces to the following linear system:

$$
(n-1) \gamma_{k}-\sum_{i \neq k} \gamma_{i}=0, \quad k=2, \ldots, n,
$$

with the unique solution $\gamma_{2}=\ldots=\gamma_{n}=0$. By (4.12), it follows that $\lambda_{i}=\lambda:=\lambda_{1}$ for all $i \in N$.

Step 3. Assume that $n \geq 2$. We now prove that $\lambda \in[0,1]$. For this, fix $i \in N$ and consider the unanimity game $v_{N \backslash i}$ given by $v_{N \backslash i}(S)=1$ for $S=N$ or $S=N \backslash i$, and $v_{N \backslash i}(S)=0$ otherwise. By Axiom A9 applied to $v=v_{N \backslash i}$, we obtain $\Phi_{i}\left(v_{N \backslash i}\right) \geq 0$. On the other hand, we have

$$
\Phi_{i}^{\mathrm{Sh}}\left(v_{N \backslash i}\right)=0, \quad \Phi_{i}^{\mathrm{Sol}}\left(v_{N \backslash i}\right)=(n-1) / n^{2} .
$$

Using (4.5) with $\lambda_{i}=\lambda$, we get

$$
0 \leq \Phi_{i}\left(v_{N \backslash i}\right)=\lambda \Phi_{i}^{\mathrm{Sh}}\left(v_{N \backslash i}\right)+(1-\lambda) \Phi_{i}^{\mathrm{Sol}}\left(v_{N \backslash i}\right)=(1-\lambda)(n-1) / n^{2},
$$

hence $\lambda \leq 1$. 
Consider now the game $w$ defined by $w(N)=-1, w(N \backslash i)=-n$, and $w(S)=0$ otherwise. By Axiom A9 applied to $w, \Phi_{i}(w) \geq 0$. At the same time

$$
\Phi_{i}^{\mathrm{Sh}}(w)=(n-1) / n, \quad \Phi_{i}^{\mathrm{Sol}}(w)=0 .
$$

Thus, we have

$$
0 \leq \Phi_{i}(w)=\lambda \Phi_{i}^{\mathrm{Sh}}(w)+(1-\lambda) \Phi_{i}^{\mathrm{Sol}}(w)=\lambda(n-1) / n,
$$

hence $\lambda \geq 0$. Putting $\alpha=(1-\lambda) / \lambda$, we complete the proof for $n \geq 2$.

If $n=1$, the proof of $(\Rightarrow)$ is trivial.

\section{References}

[1] A. S. Nowak and T. Radzik, A solidarity value for n-person transferable utility games, Internat. J. Game Theory 23 (1994), 43-48.

[2] L. S. Shapley, A value for n-person games, in: Contributions to the Theory of Games II, Ann. Math. Stud., H. W. Kuhn and A. W. Tucker (eds.), Princeton University Press, Princeton, 1953, 307-317.

Andrzej S. Nowak and Tadeusz Radzik

Institute of Mathematics

Technical University of Wrocław

Wybrzeże Wyspiańskiego 37

50-370 Wrocław, Poland 